Check for updates

Cite this: Phys. Chem. Chem. Phys. 2019, 21, 4568

Received 28th November 2018 Accepted 4th February 2019

DOI: $10.1039 / c 8 c p 07311 b$

rsc.li/pccp

\section{Spectroscopic characterisation of centropolyindanes $\dagger$}

\begin{abstract}
Stewart F. Parker, (D) *a Lisha Zhong, (D) ${ }^{b}$ Marco Harig ${ }^{c}$ and Dietmar Kuck (D) ${ }^{c}$
A highly promising class of three-dimensional polyaromatic hydrocarbons comprises the centropolyindanes. The characteristic feature of these compounds is the mutual fusion of several molecules of indane along the saturated $\mathrm{C}-\mathrm{C}$ bonds of their cyclopentane rings. Among the polycyclic aromatic hydrocarbons, the centropolyindanes are special because of the saturated core of $\mathrm{sp}^{3}$-hybridised carbon atoms embedded in a three-dimensional environment of aromatic building blocks. While the centropolyindanes and their numerous derivatives have been studied in detail by NMR spectroscopy, mass spectrometry and X-ray diffraction, investigation of their vibrational features, and especially those of the neopentane core present in most cases, have not been performed so far. In the present paper, we report the first systematic study of a set of centropolyindanes by vibrational spectroscopy, using inelastic neutron scattering (INS), infrared and Raman spectroscopies.
\end{abstract}

\section{Introduction}

In the past decades, the combination of the fundamental structural tool-kit of organic chemistry with geometrically strict motifs has led to an enormous expansion of non-natural molecular architecture that represents a classical field of chemical research. ${ }^{1}$ This holds true in particular for ideally two-dimensional carbon frameworks based on hexagonal benzene building blocks ${ }^{2,3}$ as well as for the distorted variants, most of which contain non-hexagonal, such as five-, seven- and eight-membered ring elements, ${ }^{4-9}$ and includes tube-like ${ }^{10}$ and fully globular carbon networks. ${ }^{11}$ Other albeit less variable molecular motifs comprise linear, acetylene- and cumulenebased carbon chains. ${ }^{12}$ Strictly three-dimensional carbon-based constructs have remained relatively scarce but have started to gain increasing attention. ${ }^{13-17}$ In this context, a highly promising class of novel three-dimensional molecular motifs comprises the centropolyindanes. $^{18}$

The characteristic feature of the centropolyindane hydrocarbons is the mutual fusion of several molecules of indane (1, Chart 1) along the saturated $\mathrm{C}-\mathrm{C}$ bonds of their five-membered (cyclopentane) rings. For example, several centrotriindanes are known, such as triptindane, 2 (a tribenzo[3.3.3]propellane or

\footnotetext{
${ }^{a}$ ISIS Facility, STFC Rutherford Appleton Laboratory, Chilton, Didcot, OX11 OQX, UK. E-mail: stewart.parker@stfc.ac.uk

${ }^{b}$ Downing College, University of Cambridge, Regent Street, Cambridge, CB2 1DQ, UK

${ }^{c}$ Department of Chemistry and Center for Molecular Materials $\left(\mathrm{CM}_{2}\right)$,

Bielefeld University, 33615 Bielefeld, Germany

$\dagger$ Electronic supplementary information (ESI) available: Synthesis of compound 8, structure and spectra of compounds 1-10, comparisons of observed and calculated infrared and INS spectra. See DOI: $10.1039 / \mathrm{c} 8 \mathrm{cp} 07311 \mathrm{~b}$
}

"monofuso-centrotriindane"), ${ }^{13,19,20}$ the angularly fused difusocentrotriindane $4,{ }^{21}$ and tribenzotriquinacene 3 (a trifuso-centrotriindane). ${ }^{22,23}$ Fenestrindane, $6,{ }^{24}$ represents one of the two possible regular centrotetraindanes. ${ }^{18}$ As an extreme, six indane units can be merged about a common neopentane core, generating centrohexaindane, which has been addressed as a "Cartesian" hydrocarbon due to the strictly orthogonal orientation of its six benzene units in space. ${ }^{25}$ In recent years, decisive progress has been made to merge the particular structural motifs of tribenzotriquinacene (3) and fenestrindane (6) with genuine benzenoid frameworks. ${ }^{26,27}$

Among the polycyclic aromatic hydrocarbons, the centropolyindanes are special because of the saturated, $\mathrm{sp}^{3}$-hybridised core embedded in a three-dimensional environment of aromatic building blocks. While these three-dimensional hydrocarbons and their numerous derivatives have been studied in detail by NMR spectroscopy, mass spectrometry and X-ray diffraction, ${ }^{28}$ investigation of their vibrational features, and especially those of the neopentane core, have not been performed so far. In the present paper, we report the first systematic study of a set of centropolyindanes by vibrational spectroscopy, using inelastic neutron scattering (INS), infrared and Raman spectroscopies. The nine centropolyindanes, 2-10, investigated here are displayed in Chart 1, along with their structural parent hydrocarbon, indane (1).

\section{Experimental}

\section{Materials}

Indane (Aldrich, 95\%) was used as received. Triptindane $(2),{ }^{20}$ tribenzotriquinacene $(3),{ }^{22}$ difuso-centrotriindane $(4),{ }^{21}$ 


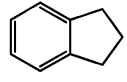

1

$\mathrm{C}_{9} \mathrm{H}_{10}, \mathrm{C}_{s}$

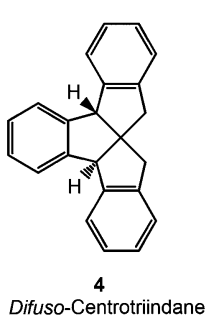

$\mathrm{C}_{23} \mathrm{H}_{18}, \mathrm{C}_{1}$

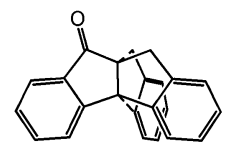

Triptindan-9-one

$\mathrm{C}_{23} \mathrm{H}_{16}, \mathrm{C}_{1}$

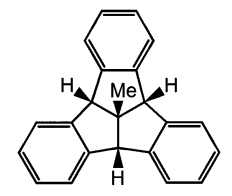

9

12d-Methyltribenzotriquinacene

$\mathrm{C}_{23} \mathrm{H}_{18}, \mathrm{C}_{3 v}$

R3m, QOWNUL $[30,41]$

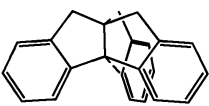

Triptindane

$\mathrm{C}_{23} \mathrm{H}_{18}, \mathrm{C}_{3}$

$P \overline{1}$, EVAYAD [40]

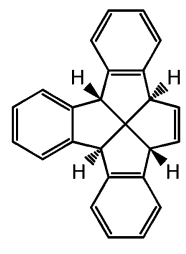

5 Tribenzo[5.5.5.5]fenestrene $\mathrm{C}_{25} \mathrm{H}_{18}, \mathrm{C}_{1}$

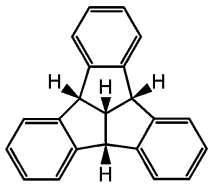

Tribenzotriquinacene (TBTQ)

$\mathrm{C}_{22} \mathrm{H}_{16}, \mathrm{C}_{3 v}$

R3c, DEYQIJ [41]

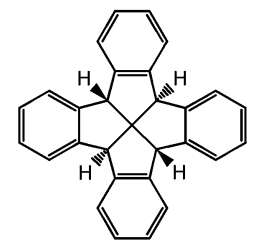

6

Fenestrindane

$\mathrm{C}_{29} \mathrm{H}_{20}, \mathrm{~S}_{4}$ $P \overline{1}$, FARLEP [24]
Chart 1 Structures of the compounds studied. Below the sample numbers, the chemical name, the empirical formula and the molecular point group are given. If the solid-state structure has been determined, the space group, followed by the Cambridge Structural Database ${ }^{29}$ refcode and the reference to the structure are also presented. Ball-and-stick representations of the structures are given in Fig. S1-S6, S13, S15, S17 and S19 of the ESI. $\dagger$

tribenzo[5.5.5.5]fenestrene (5), ${ }^{24}$ fenestrindane (6), ${ }^{24}$ triptindan-9one (7), ${ }^{20} 12 d$-methyltribenzotriquinacene $(9)^{21}$ and $4 b, 8 b, 12 b, 12 d$ tetramethyltribenzotriquinacene $(\mathbf{1 0})^{30}$ were synthesised as described in the literature. ${ }^{18}$ The synthesis of 1,4-dimethoxytriptindane (8) was performed as shown in Scheme 1. The details are given in the ESI. $\dagger$

\section{Vibrational spectroscopy}

Inelastic neutron scattering (INS) spectra were recorded at less than $20 \mathrm{~K}$ using TOSCA ${ }^{31}$ at ISIS (http://www.isis.stfc.ac.uk/). The spectra are available at the INS database: http://wwwisis2. isis.rl.ac.uk/INSdatabase/. Infrared spectra were recorded using a Bruker Vertex70 FTIR spectrometer, over the range 100 to $4000 \mathrm{~cm}^{-1}$ at $4 \mathrm{~cm}^{-1}$ resolution with a DLaTGS detector using 64 scans and the Bruker Diamond ATR. Variable temperature infrared spectra were recorded for some samples using a Specac Golden Gate accessory. The use of the ultra-wide range beam splitter enabled the entire spectral range to be recorded
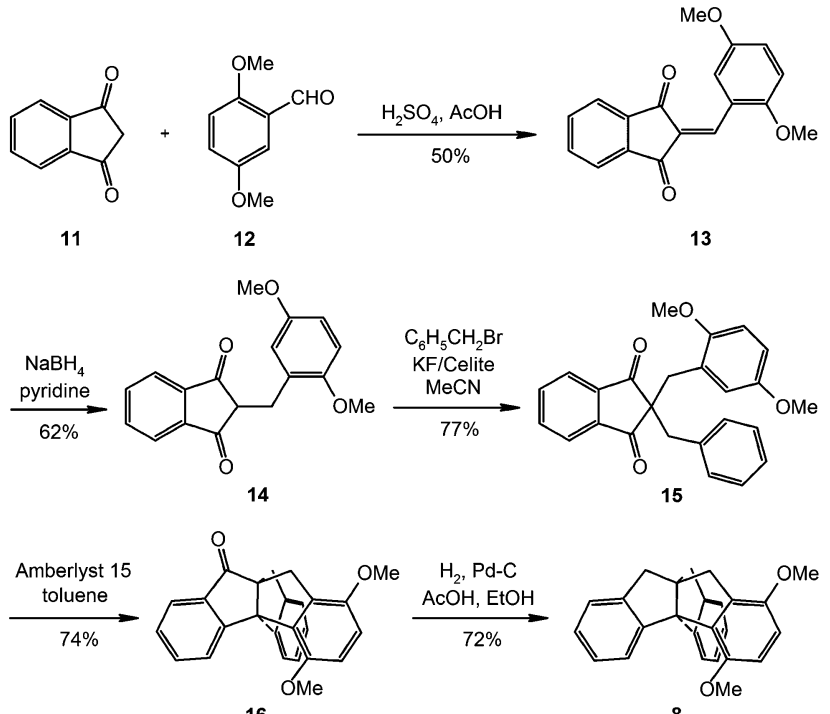

Scheme 1 Synthesis of 1,4-dimethoxytriptindane (8).

without the need to change beam splitters. The spectra have been corrected for the wavelength-dependent variation in path length using the Bruker software. FT-Raman spectra were recorded with a Bruker MultiRam spectrometer using $1064 \mathrm{~nm}$ excitation, $4 \mathrm{~cm}^{-1}$ resolution, $500 \mathrm{~mW}$ laser power and 64 scans. Variable temperature Raman spectra were recorded using $785 \mathrm{~nm}$ excitation and a Renishaw InVia spectrometer that has been previously described. ${ }^{32}$ For both Raman instruments, the low wavenumber limit was $\sim 50 \mathrm{~cm}^{-1}$, as determined by the laser line rejection filter.

\section{Computational studies}

Isolated molecule calculations were carried out with Gaussian 03 and Gaussian 09. ${ }^{33}$ In both cases, the 6-311G(d) basis set was used with the B3LYP hybrid functional. Solid state calculations used the plane wave pseudopotential-based program CASTEP $^{34,35}$ with the inclusion of the Tkatchenko and Scheffler $^{36}$ dispersion correction for the calculation of the vibrational transition energies and their intensities. The generalized gradient approximation Perdew-Burke-Ernzerhof functional was used in conjunction with optimized normconserving pseudopotentials. All of the calculations were converged to better than $|0.0035| \mathrm{eV} \AA^{-1}$. After geometry optimization, the vibrational spectra were calculated in the harmonic approximation using density functional perturbation theory. Both Gaussian and CASTEP generate the vibrational eigenvalues and eigenvectors, which allows visualization of the modes within GaussView ${ }^{37}$ and Materials Studio (http://accelrys.com/products/collaborative-science/ biovia-materials-studio/) respectively. It is also the information needed to calculate the INS spectrum using the program ACLIMAX. ${ }^{38}$ Infrared and Raman spectra were generated from the Gaussian output using either GaussView or GaussSum. ${ }^{39}$ We emphasize that the transition energies have not been scaled. 


\section{Results and discussion}

The structures of the compounds studied are shown in Chart 1. Ball-and-stick representations are given in Fig. S1-S6, S13, S15, S17 and S19 of the ESI. $\dagger$ For some of the compounds, the structure is known from X-ray crystallography $\left(2,{ }^{40} 3,{ }^{41} 6^{24}\right.$ and $9^{30,41}$ ), the remainder have been characterised by solution NMR spectroscopy and mass spectrometry. This group of compounds was chosen so as to examine: (i) how the progressive addition of indane (1) sub-units to the core $(\mathbf{2}, \mathbf{3}, \mathbf{4}, \mathbf{5}, \mathbf{6})$ and (ii) substitution of the indane framework $(2,7,8)$ and substitution at the central carbons $(3,9,10)$ modifies the spectra.

Fig. 1-3 show the INS, infrared and FT-Raman spectra of a series of compounds in order of increasing complexity $(\mathbf{1}, \mathbf{2}, \mathbf{3}$, $\mathbf{4}, \mathbf{5}, \mathbf{6})$. The three types of spectra for each compound are shown in Fig. S1-S6 (ESI $\dagger$ ).

While the spectra are clearly very rich, each technique shows a remarkable similarity between the different molecules. In the INS spectra (Fig. 1), the overall pattern is similar in the $400-1600 \mathrm{~cm}^{-1}$ range, in the infrared spectra (Fig. 2), strong bands are seen at $\sim 450,750$ and $1480 \mathrm{~cm}^{-1}$, and in the Raman spectra (Fig. 3), strong modes occur at $\sim 150,750,1020$ and $1600 \mathrm{~cm}^{-1}$. This suggests that the vibrational spectra are largely characteristic of indane. While it is perhaps unsurprising that the aromatic modes are largely unchanged, the very different central framework of the molecules might have been expected to be reflected in the spectra. The approach that has been adopted was to carry out a detailed assignment of the parent molecule, indane (1), and then to examine the differences between the systems.

\section{Indane (1)}

Indane (1) has two possible conformations of the C5 ring: planar $\left(C_{2 \mathrm{v}}\right)$ and buckled $\left(C_{\mathrm{s}}\right)$. It is formally a cyclopentene

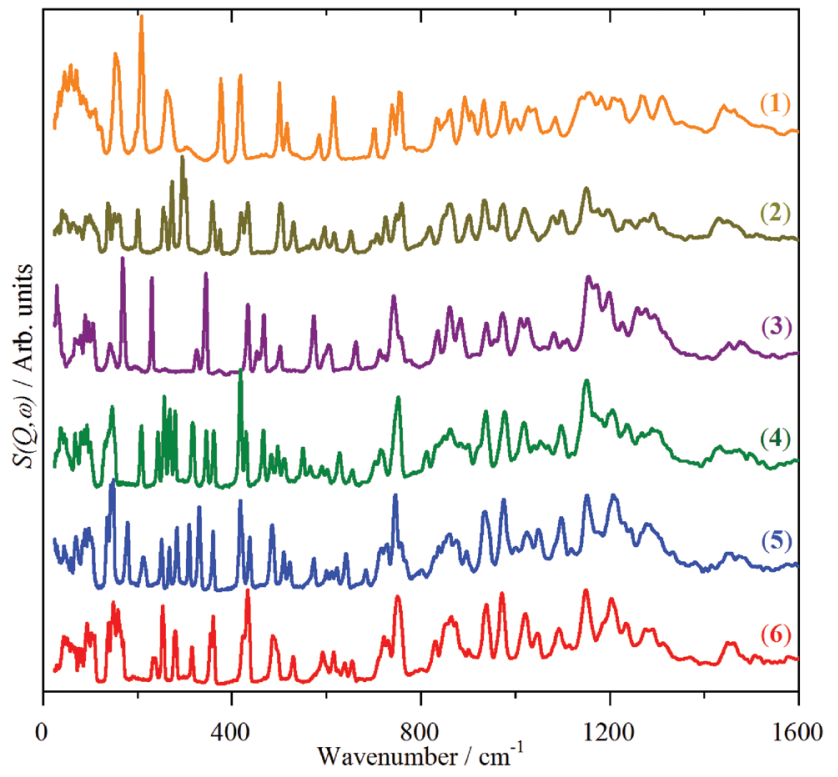

Fig. 1 INS spectra from top to bottom of: solid indane (1), triptindane (2), tribenzotriquinacene (3), difuso-centrotriindane (4), tribenzo[5.5.5.5]fenestrene (5) and fenestrindane (6).

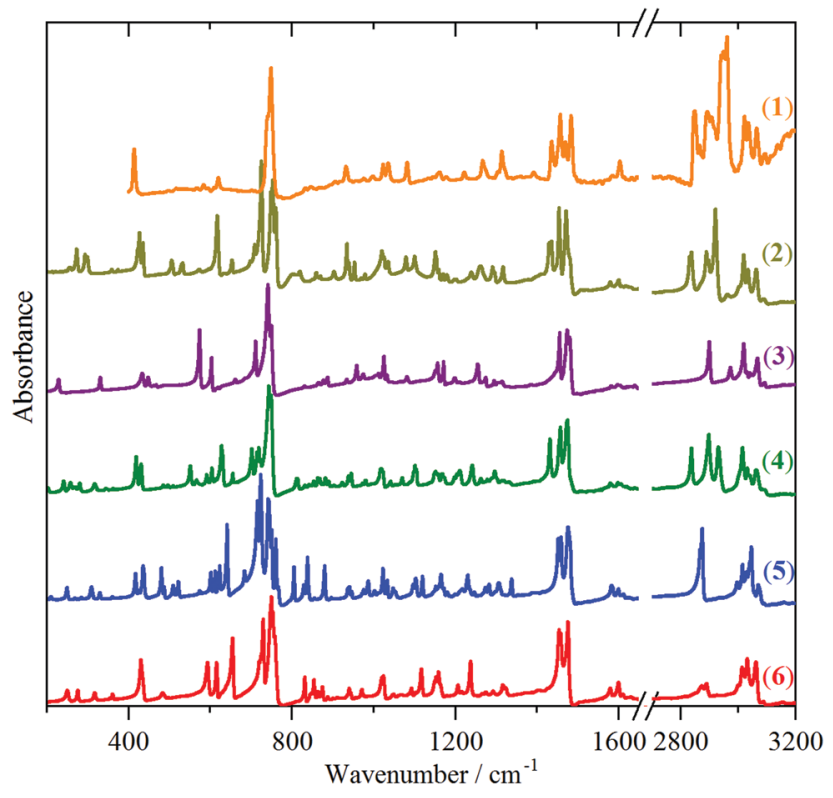

Fig. 2 Infrared spectra from top to bottom of: solid indane (1), triptindane (2), tribenzotriquinacene (3), difuso-centrotriindane (4), tribenzo[5.5.5.5]fenestrene (5) and fenestrindane (6).

derivative and the parent molecule is known to be puckered, ${ }^{42,43}$ i.e., to have $C_{\mathrm{s}}$ symmetry. Further, while the crystal structure of indane itself is not known, that of many of its derivatives are known and these all exhibit a puckered C5 ring. For example, in 4- and 5-nitroindane ${ }^{44}$ the $\mathrm{CH}_{2}$ group is $5.0^{\circ}$ and $12.7^{\circ}$ out of the ring and in trans,trans-1,2,3-triphenylindane ${ }^{45}$ it is $16.9^{\circ}$ out of the ring. An isolated molecule, i.e. gas phase, calculation of the planar $C_{2 \mathrm{v}}$ structure found one imaginary mode that corresponded to movement of the $\mathrm{CH}_{2}$ group out-of-the plane. Comparison of the calculated isolated molecule buckled $\left(C_{\mathrm{s}}\right)$ structure with the gas phase structure ${ }^{46}$ shows good agreement.

Fig. 4 compares the experimental solid state INS spectrum and the calculated isolated (i.e. gas phase) molecule INS spectrum of indane. The isolated molecule does not include the lattice modes (the intense broad band centered at $\sim 100 \mathrm{~cm}^{-1}$ in Fig. 4a) but in the internal mode region above $150 \mathrm{~cm}^{-1}$, it can be seen that the agreement is excellent. Fig. S7 $(\mathrm{ESI} \dagger)$ shows similarly good agreement for the infrared spectra, with the exception of the $\mathrm{C}-\mathrm{H}$ stretch modes which are, as is the usual case, calculated at too high an energy. This is because the calculation assumes a harmonic potential and the real system is anharmonic. ${ }^{47}$

Spectroscopic studies of indane have been dominated by attempts to determine the puckering potential in the gas phase. ${ }^{48-50}$ The infrared and Raman spectra and their assignment for the liquid phase have been reported; ${ }^{51}$ there are no data for the solid state. Table S1 (ESI $\dagger$ ) lists the observed and calculated transition energies and their assignment. These are largely in agreement with the only previous work, ${ }^{51}$ which was based on an empirical force field. The disagreements are mainly in the low energy region, $<500 \mathrm{~cm}^{-1}$, where the infrared and Raman bands are weak, whereas the deformations result in 

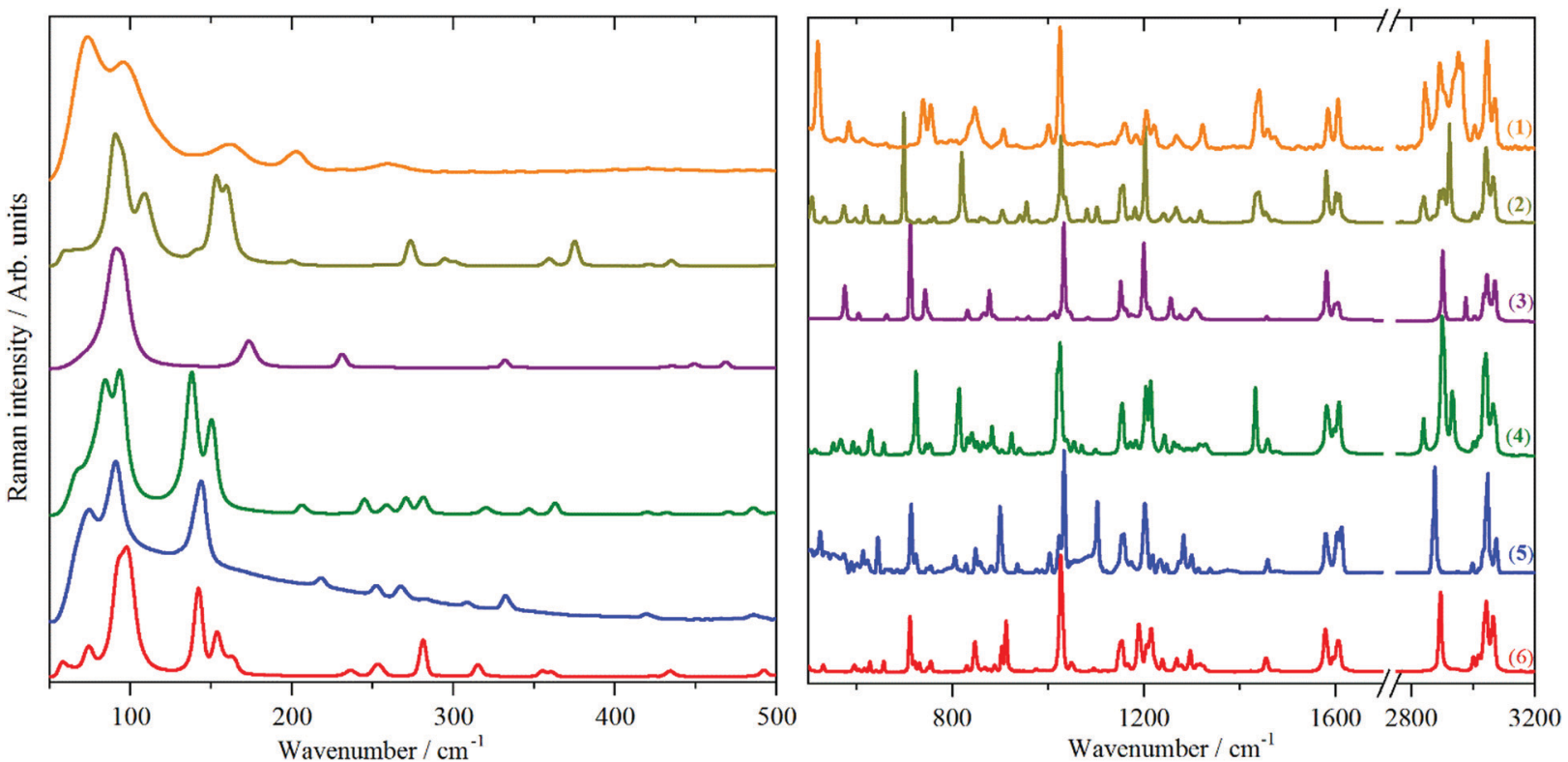

Fig. 3 FT-Raman spectra from top to bottom of: solid indane (1), triptindane (2), tribenzotriquinacene (3), difuso-centrotriindane (4), tribenzo[5.5.5.5]fenestrene (5) and fenestrindane (6). The right-hand side set of spectra $\left(500-3200 \mathrm{~cm}^{-1}\right)$ are $\times 2, \times 3, \times 4, \times 5, \times 4, \times 4$ respectively, ordinate expanded relative to the left-hand side set $\left(50-500 \mathrm{~cm}^{-1}\right)$.

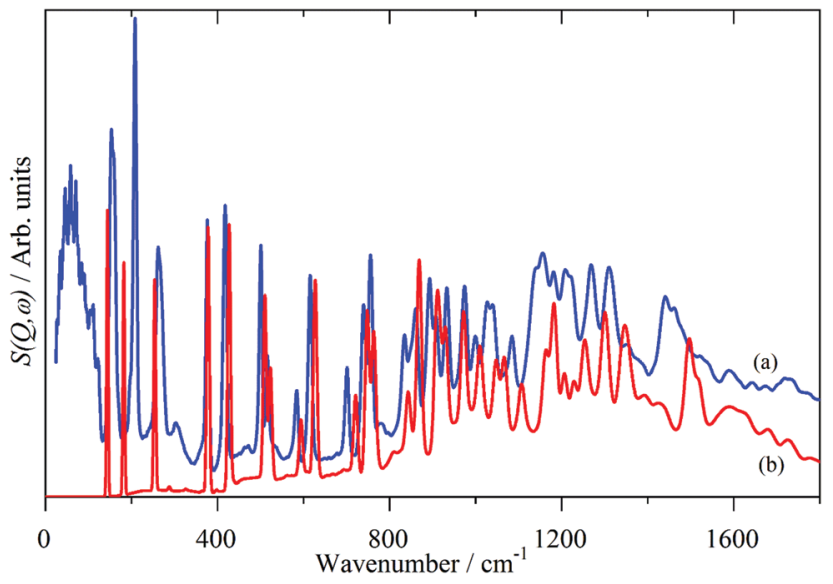

Fig. 4 Comparison of INS spectra of indane (1): (a) experimental in the solid state and (b) calculated for the isolated molecule. The bands below $150 \mathrm{~cm}^{-1}$ in (a) are the lattice modes and are outside of the scope of the calculation shown in (b).

large amplitude motions of the hydrogen atoms and hence strong INS modes. It is noteworthy that the transition energies of the phenylene $\left(\mathrm{C}_{6} \mathrm{H}_{4}\right)$ modes are typical of those found for ortho-disubstituted benzenes. ${ }^{52,53}$

\section{Comparison of the spectra}

As crystal structures are not available for the whole set of the compounds, we anticipated that in the absence of significant intermolecular interactions, the isolated molecule approximation should provide a good description of the vibrational spectra. To enable comparisons, this was used for all of the molecules. Tribenzotriquinacene (3) is the simplest of the centropolyindanes studied here, consisting of three fused indane sub-units, sharing the common apical carbon of the five-membered rings. This provides a test of this assumption as the crystal structure is known. ${ }^{41}$ It consists of stacks of molecules aligned along the $c$-axis, with the closest intermolecular distance being $\mathrm{H} \cdots \mathrm{H}$ contacts at 2.220 A. Fig. 5 compares the experimental INS spectrum with those calculated using the isolated molecule approximation (Gaussian) and the full periodic calculation (CASTEP). It can be seen that both methods are in good agreement with the data and while the CASTEP calculation produces a better overall match, the differences are small, justifying the use of isolated molecule calculations.

For molecular solids such as these it might be expected that inclusion of long-range interactions would be required to obtain good agreement between experiment and theory. We have investigated this possibility for both intermolecular and intramolecular consequences. As explained further in the ESI, $\dagger$ the effect of the inclusion of the long-range interactions via the Tkatchenko and Scheffler ${ }^{36}$ (TS) correction is very small for the systems studied here.

Fig. S8-S12 (ESI $\dagger$ ) compare the calculated and observed INS and infrared spectra for the centropolyindanes 2-6. In all cases the agreement is acceptable and enables assignments to be made. As suggested by the comparison with indane, the strong infrared features at 450, 750 and $1480 \mathrm{~cm}^{-1}$ are assigned as phenylene modes (the Wilson ${ }^{52}$ labelling of the parent benzene modes is given in brackets): out-of-plane ring deformation (16b), C-H out-of-plane bend (11) and C-C stretch (19a), respectively. Similarly, for the Raman spectra, the strong mode at $\sim 1020 \mathrm{~cm}^{-1}$ is the phenylene $\mathrm{C}-\mathrm{H}$ in-plane bend $(18 \mathrm{~b})$. All of the centropolyindanes have a very strong Raman mode (note the difference in ordinate scales between the left and 


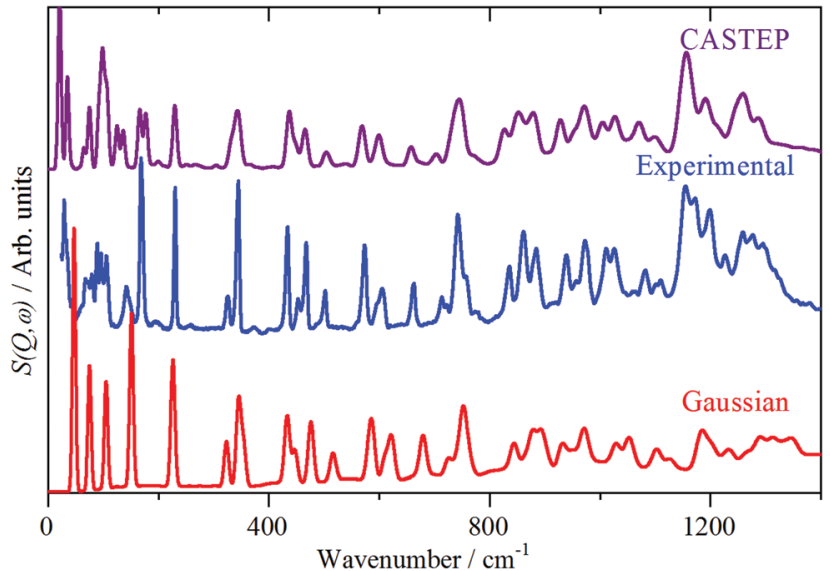

Fig. 5 Comparison of INS spectra of tribenzotriquinacene (3): experimental spectrum (middle) and those calculated using the isolated molecule approximation (bottom, Gaussian) and from a full periodic calculation (top, CASTEP).

right sides of Fig. 3) at $\sim 100 \mathrm{~cm}^{-1}$, and compounds $2, \mathbf{4 , 5}$ and 6, but neither indane (1) nor tribenzotriquinacene (3) have a strong mode at $\sim 150 \mathrm{~cm}^{-1}$. (The same modes are also seen in the INS spectra.) Visualisation of the modes shows that the $100 \mathrm{~cm}^{-1}$ mode is related to the indane mode at $\sim 160 \mathrm{~cm}^{-1}$, (C9-C1 + C4-C3 in-phase torsion), that has shifted down in energy because of the replacement of hydrogen atoms by the heavier indane moieties. This fragment is common to all the molecules and thus appears in all their spectra. In contrast, the $\sim 150 \mathrm{~cm}^{-1}$ mode involves the central neopentane core and is formally derived from one component of $\nu_{19}$, a triply degenerate $\mathrm{C}-\mathrm{C}-\mathrm{C}$ bending motion. ${ }^{54}$ This explains why it is only seen in compounds 2, 4, 5 and 6: only these have the fully developed neopentane core. The modes are shown in Fig. 6 for fenestrindane (6), together with a comparison of the calculated spectra for the isolated molecule $(Z=1)$ and the primitive cell $(Z=2)$. It can be seen that for these very low energy modes there is a small, but significant, influence of the intermolecular interactions.

At the opposite end of the spectra, the $\mathrm{C}-\mathrm{H}$ stretch region is remarkably simple (Fig. 7). In the aromatic region, $>3000 \mathrm{~cm}^{-1}$, for both the Raman and the infrared spectra the pattern found for indane is largely replicated, consisting of two and three bands, respectively. This indicates that, in all the samples, the phenylene groups of the indane residues behave as independent entities, there is little or no coupling between them. The aliphatic region shows more variety, but this is understandable because of the different types of $\mathrm{C}-\mathrm{H}$ groups present. Thus indane (1) has three methylene groups, which, as the spectra indicate, are coupled (since there are more than the two modes expected for isolated oscillators: the symmetric, $\sim 2850 \mathrm{~cm}^{-1}$, and asymmetric, $\sim 2940 \mathrm{~cm}^{-1}, \mathrm{H}-\mathrm{C}-\mathrm{H}$ stretch modes). Triptindane (2) also has three methylene groups, but these are separated from each other by the central carbon atom. Nonetheless, the spectrum resembles that of indane. Compounds $\mathbf{3 , 5}$ and $\mathbf{6}$ each contain four bridgehead methine groups but only show a single band at $\sim 2890 \mathrm{~cm}^{-1}$, in both the Raman and infrared

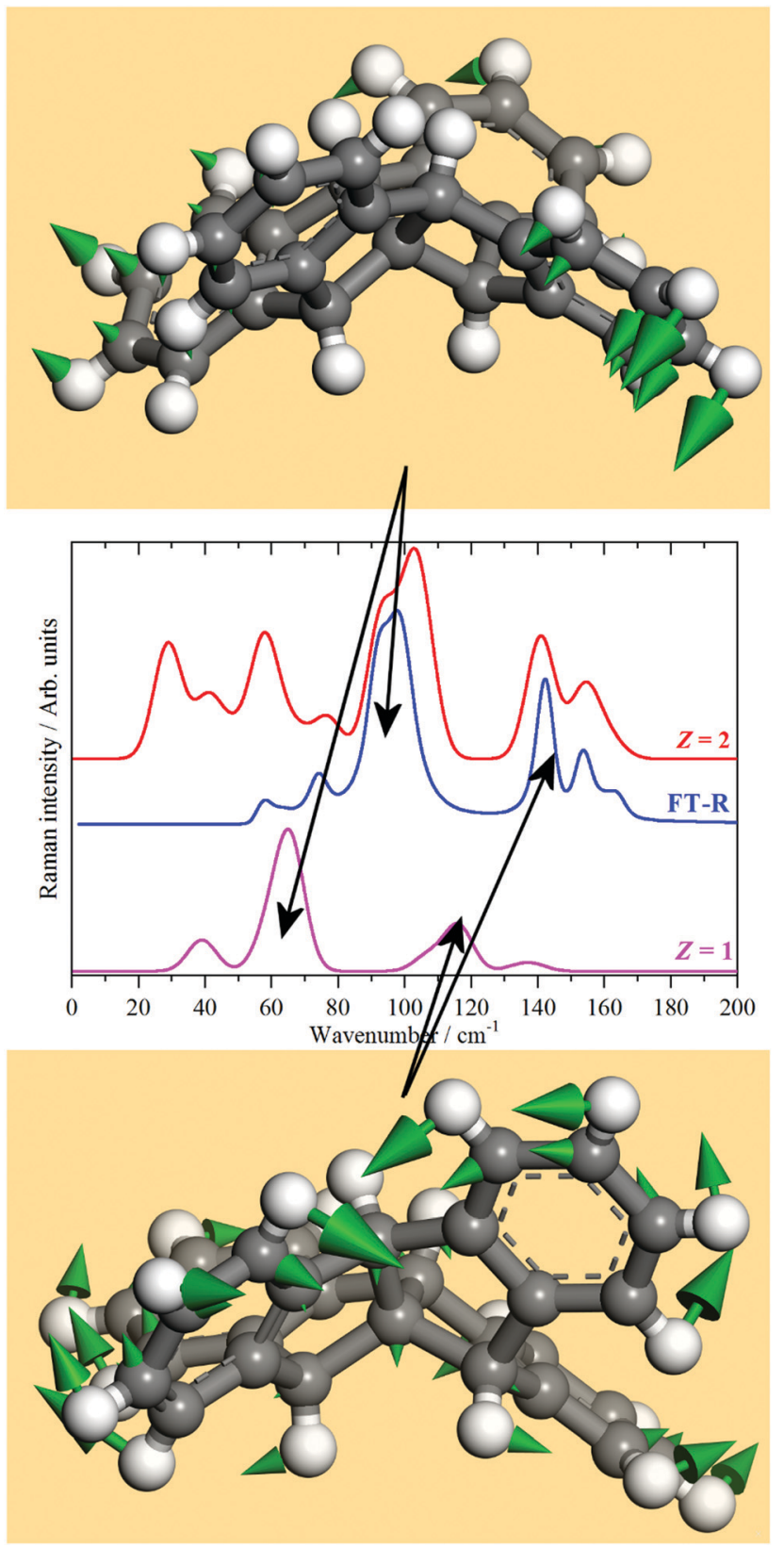

Fig. 6 Comparison of Raman spectra of fenestrindane (6) in the low energy region: experimental (FT-R) spectrum and that calculated using the isolated molecule approximation $(Z=1)$ and from a full periodic calculation $(Z=2)$. The top part shows the torsional mode and the lower part the neopentane-derived mode. Note the different displacements of the indane rings.

spectra. Difuso-centrotriindane 4 bears two methine groups and two methylene groups; thus the spectrum shows the methylene doublet at 2840 and $2930 \mathrm{~cm}^{-1}$, and the methine band at $2900 \mathrm{~cm}^{-1}$.

\section{The C-C stretch modes}

Potentially, the most interesting modes are those that involve carbon atoms that are common to two or more indane sub-units because these reflect the strength of the bonding between the 


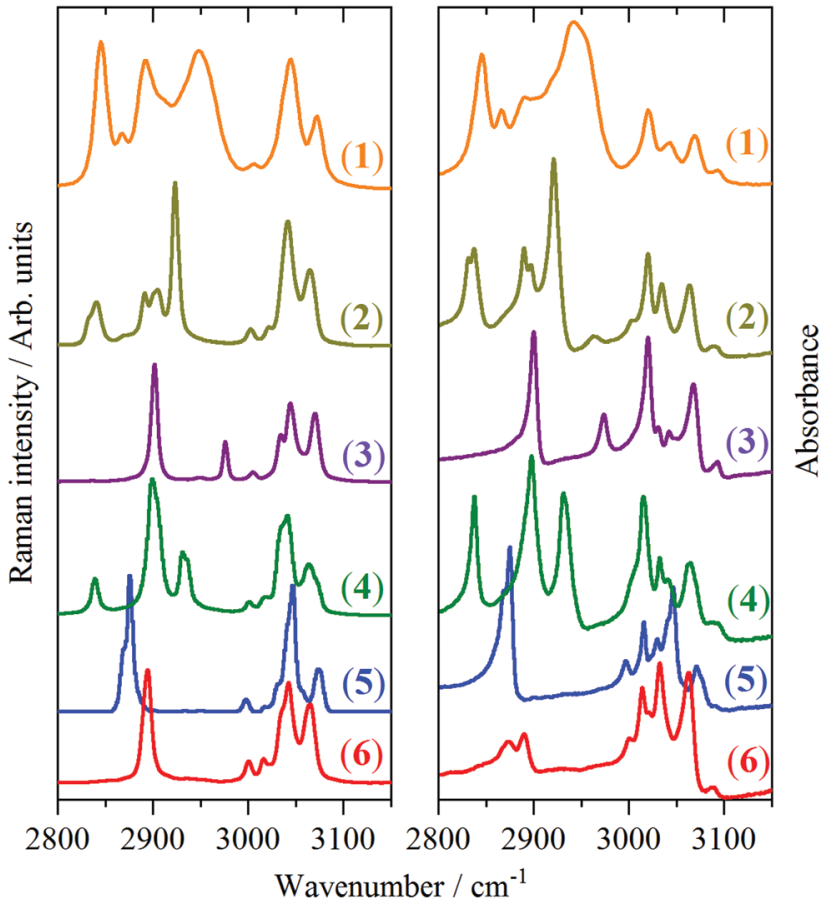

Fig. 7 Raman (left) and infrared (right) spectra in the $\mathrm{C}-\mathrm{H}$ stretch region from top to bottom, of: solid indane (1), triptindane (2), tribenzotriquinacene (3), difuso-centrotriindane (4), tribenzo[5.5.5.5]fenestrene (5) and fenestrindane (6).

indane sub-units. However, as we have already seen, the spectra are dominated by the ortho-phenylene modes and the aliphatic framework does not contribute strong modes (except for the low energy deformation mode described previously). The DFTcalculated INS spectra provide a means to access the aliphatic $\mathrm{C}-\mathrm{C}$ stretch modes. By setting the cross sections of all the atoms to zero except those of interest, the resulting "pseudospectra" only contain modes where the atom(s) of interest have significant motion. Fig. 8 shows the results of this exercise where only the carbon atom that is common to the most indane sub-units has a non-zero cross section. Thus for compounds $\mathbf{2}$, 3,4 and 5 the common carbon atom links three indane sub-units and for compound $\mathbf{6}$ it links four indane sub-units. For tribenzotriquinacene (3) this is a tertiary carbon atom, for the hydrocarbons $2, \mathbf{4 , 5}$ and $\mathbf{6}$ it is a quaternary carbon atom.

From Fig. 8, it can be seen that there are three regions where there is significant motion of the atom of interest: $450-650 \mathrm{~cm}^{-1}$, $1000-1150 \mathrm{~cm}^{-1}$ and $1250-1400 \mathrm{~cm}^{-1}$. Note that the pseudospectra only report modes where there is motion of the atom, not whether it is the driving force of the mode. Visualisation of the modes shows that the intensity in the $1250-1400 \mathrm{~cm}^{-1}$ region is a consequence of the requirement for a normal mode that the centre of mass is invariant, thus atoms can move simply to satisfy this criterion, even though there is no change in bond lengths or angles that involve the atoms. A similar situation is found for the modes at $450-650 \mathrm{~cm}^{-1}$, although in the case of compounds 2 and 3 the mode around $550 \mathrm{~cm}^{-1}$ does include contributions from $\mathrm{C}-\mathrm{C}-\mathrm{C}$ bending modes involving the atom of interest. The modes at $1000-1150 \mathrm{~cm}^{-1}$ do have

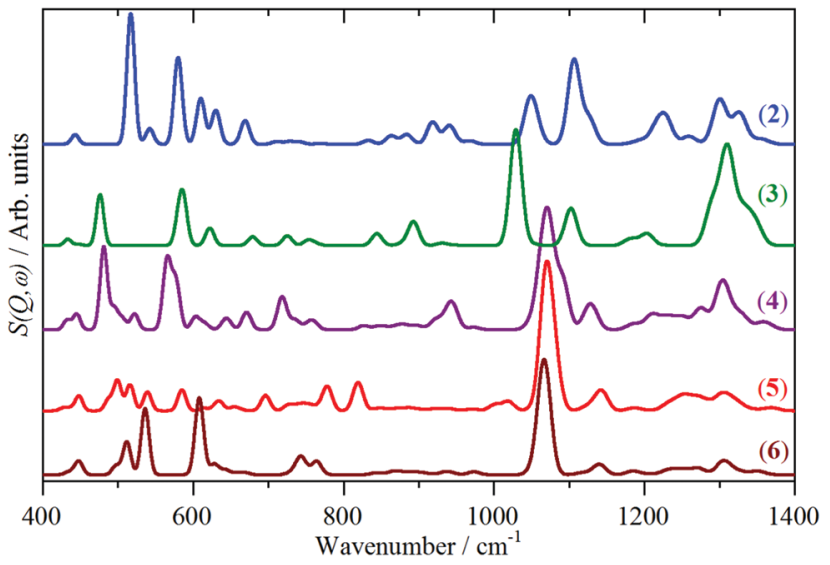

Fig. 8 INS pseudo-spectra generated from the DFT calculations showing only the modes that involve motion of the central carbon atom(s). From top to bottom: triptindane (2), tribenzotriquinacene (3), difusocentrotriindane (4), tribenzo[5.5.5.5]fenestrene (5) and fenestrindane (6).

large components of motion that contain $\mathrm{C}-\mathrm{C}$ stretch modes of the central carbon atom. In triptindane (2), the C-C stretch of the central carbon atoms (the two common to all three indane sub-units) is calculated at $1047 \mathrm{~cm}^{-1}\left(1036 \mathrm{~cm}^{-1}\right.$ observed). The similarity of the transition energy in all cases indicates that the bonding is also of similar strength; the additional indane unit makes little difference to the transition energy for fenestrindane (6). What is apparent is that the local symmetry around the carbon atom is important: for compounds 2 and $\mathbf{3}$ it is trigonal $\left(C_{3 \mathrm{v}}\right)$ and this gives two modes, for compounds $\mathbf{4}, \mathbf{5}$ and 6 it is tetrahedral $\left(T_{\mathrm{d}}\right)$. This results in a triply degenerate asymmetric stretch mode $\left(T_{2}\right)$ and a non-degenerate symmetric $\left(A_{1}\right) \mathrm{C}-\mathrm{C}$ stretch mode. While the local symmetry is $T_{\mathrm{d}}$, the molecular symmetry is lower and this lifts the degeneracy of the $T_{2}$ mode, so for $\mathbf{6}$, the mode at $1065 \mathrm{~cm}^{-1}$ actually consists of three components, calculated at 1063, 1069 and $1070 \mathrm{~cm}^{-1}$. For 4, 5 and 6 the $A_{1}$ mode is found at 671,661 and $657 \mathrm{~cm}^{-1}$ respectively. This is either very weak or absent from the spectra in Fig. 8 because in $T_{\mathrm{d}}$ symmetry the mode does not involve displacement of the central atom. For comparison, in neopentane $e^{54}$ the C-C stretch modes occur at $924\left(T_{2}\right)$ and $733 \mathrm{~cm}^{-1}\left(A_{1}\right)$.

\section{The effect of substitution}

The compounds investigated also enable insights into how substitution of the indane framework (2,7 and 8) and substitution at the central carbons $(\mathbf{3}, \mathbf{9}$ and $\mathbf{1 0})$ modifies the spectra.

Substitution of the indane framework. Fig. 9 shows the infrared spectra of triptindane (2), triptindan-9-one (7) and 1,4-dimethoxytriptindane (8). For the derivatives 7 and $\mathbf{8}$, the corresponding INS and FT-Raman spectra and their comparison to the calculated spectra are shown in Fig. S13-S16 (ESI $\dagger$ ). In compounds 7 and 8 only one and two carbon atoms, respectively, of 23 in the parent triptindane (2) are substituted; therefore, the overall effect on the spectra might be expected to be small. The profiles in all three cases are broadly similar, again reflecting that the spectra are largely determined by the aromatic rings. However, the substituents are clearly present, 
as evidenced by the strong carbonyl band at $1706 \mathrm{~cm}^{-1}$ in ketone 7 and the strong peaks at 1077, 1089, $1251 \mathrm{~cm}^{-1}$ and the increased width of the modes at $\sim 1450 \mathrm{~cm}^{-1}$ in the dimethoxy compound 8.

The carbonyl peak position in 7 is unusual: in cyclopentanone it occurs at $1746 \mathrm{~cm}^{-1}$, well above the usual range of $1710-1720 \mathrm{~cm}^{-1}$ for dialkyl ketones; the difference is ascribed to the effects of ring strain. ${ }^{53}$ More generally, alkyl-aryl ketones show $\mathrm{C}=\mathrm{O}$ stretch bands in the range $1680-1690 \mathrm{~cm}^{-1} \cdot{ }^{55}$ Thus aryl substitution decreases the carbonyl transition energy by $\sim 30 \mathrm{~cm}^{-1}$, which is consistent with the carbonyl transition energy of 1-indanone being $1715 \mathrm{~cm}^{-1}$. The further small

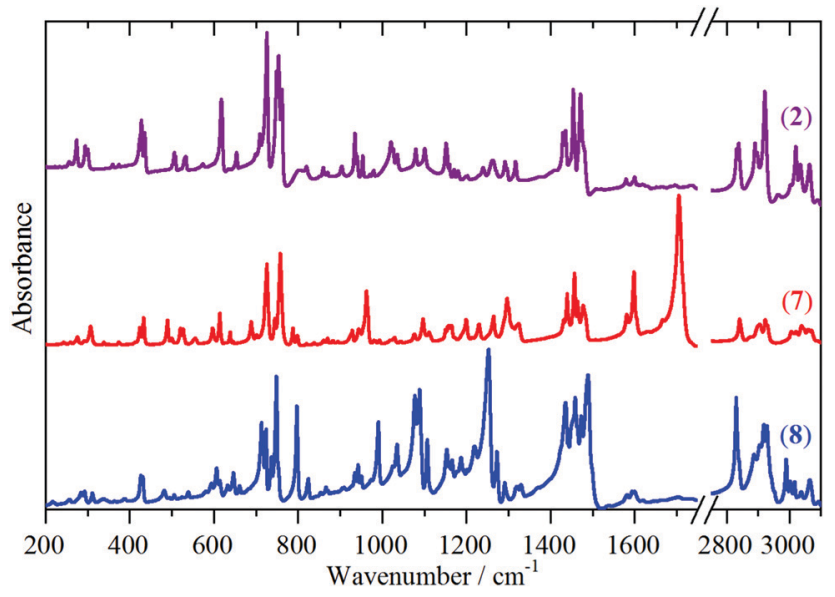

Fig. 9 Infrared spectra of triptindane (2), triptindan-9-one (7) and 1,4-dimethoxytriptindane (8). decrease must be the result of a long-range interaction with the two other indane rings, since in benzophenone the carbonyl transition energy is $1660 \mathrm{~cm}^{-1}$. That this is an electronic effect and not a ring strain effect is demonstrated by the $\mathrm{C}-\mathrm{C}(=\mathrm{O})-\mathrm{C}$ angles in cyclopentanone, ${ }^{56} 1$-indanone ${ }^{57}$ and triptindan-9-one (7) being $108.3 / 109.1^{\circ}$ (there are two independent molecules in the unit cell), $107.5^{\circ}$ and $107.3^{\circ}$ (calculated) respectively, i.e. essentially the same, but showing a $40 \mathrm{~cm}^{-1}$ difference in transition energy.

The new peaks at $1077,1089,1251 \mathrm{~cm}^{-1}$ and the increased width of the modes at $\sim 1450 \mathrm{~cm}^{-1}$ in 1,4-dimethoxytriptindane (8) are all associated with the aryl-O- $\mathrm{CH}_{3}$ functionality. They are assigned as: the $\mathrm{O}-\mathrm{CH}_{3}$ stretches $\left(1077,1089 \mathrm{~cm}^{-1}\right)$, the aryl-O stretch $\left(1251 \mathrm{~cm}^{-1}\right)$ and the symmetric and asymmetric methyl deformations $\left(1435-1470 \mathrm{~cm}^{-1}\right) .{ }^{55}$

The $\mathrm{C}-\mathrm{H}$ stretch region of ketone 7 is very similar to that of the parent hydrocarbon 2 apart from a general weakening of the bands. For the dimethoxy compound $\mathbf{8}$, this region is made more complex by the addition of the symmetric and asymmetric methyl $\mathrm{C}-\mathrm{H}$ stretch modes at the usual positions $\left(\sim 2880\right.$ and $\left.2980 \mathrm{~cm}^{-1}\right){ }^{55}$

Substitution at the central carbon atoms. Fig. 10 shows the low energy part of the INS spectra and the $\mathrm{C}-\mathrm{H}$ stretch region of the FT-Raman spectra of the tribenzotriquinacenes 3, 9 and 10. (The complete INS, infrared and Raman spectra of the derivatives $\mathbf{9}$ and $\mathbf{1 0}$ and their comparison to the calculated spectra are shown in Fig. S17-S20, ESI $\dagger$ ). The most striking changes in the INS spectra occur in the low-energy region, which is where the torsional modes occur. These are usually only easily seen in the INS spectrum. Comparison of the spectra
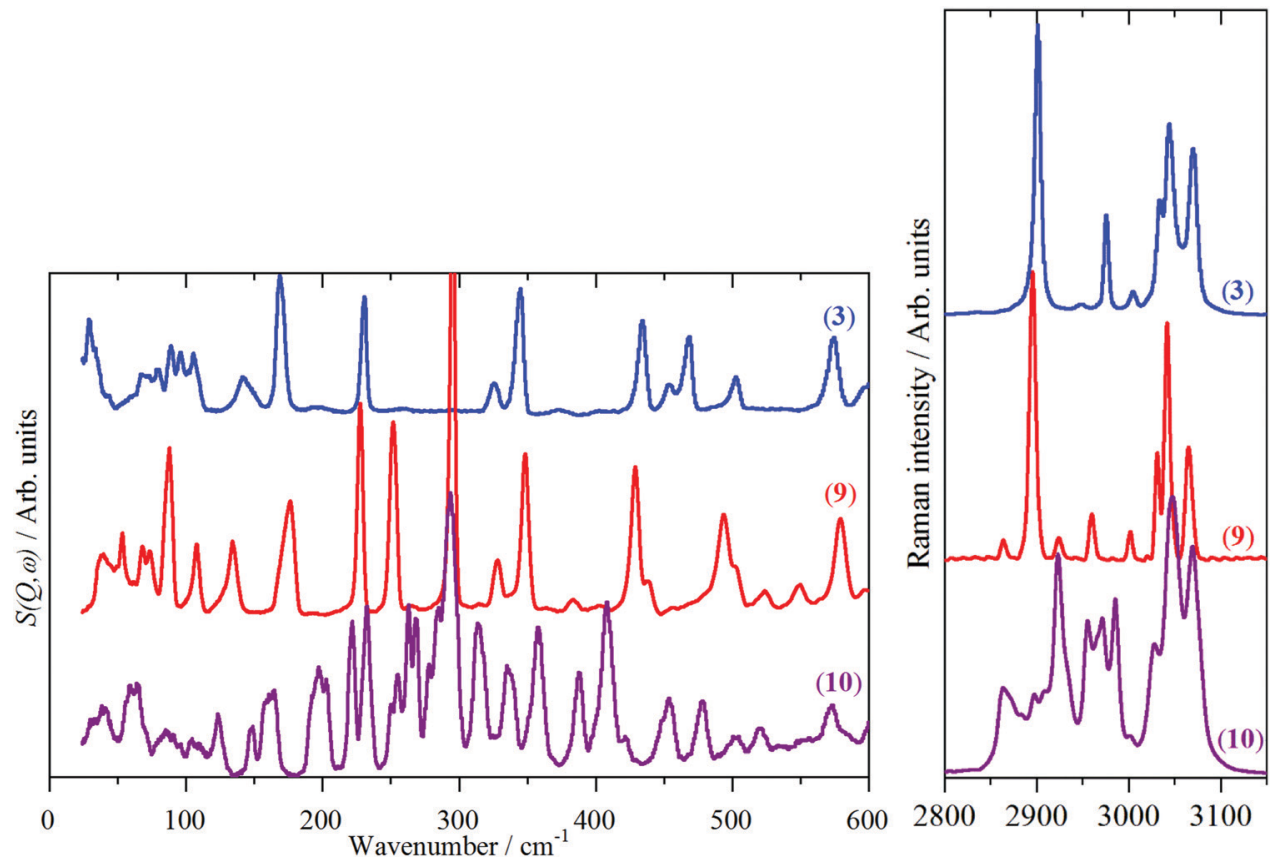

Fig. 10 Spectra of tribenzotriquinacene (3), 12d-methyltribenzotriquinacene (9) and 4b,8b,12b,12d-tetramethyltribenzotriquinacene (10). Left: INS spectra in the low energy region. Note that the INS spectra are normalised to one mole of sample and are plotted on the same ordinate scale (they are vertically displaced for clarity) so the intensities are directly comparable between the samples. Right: FT-Raman spectra in the $\mathrm{C}-\mathrm{H}$ stretch region. 
shown in Fig. 10 demonstrates that the intensities of the tribenzotriquinacene framework modes, e.g. at 350, 425 and $575 \mathrm{~cm}^{-1}$, are similar in all three cases. (Note that the spectra are normalised to one mole of sample and are plotted on the same scale, so the intensities are directly comparable between the samples.) The most striking feature in the spectrum of $12 d$-methyltribenzotriquinacene 9 in Fig. 10 is the enormously strong mode at $296 \mathrm{~cm}^{-1}$. This is assigned to the methyl torsion (and confirmed by DFT), whereas the strong mode at $252 \mathrm{~cm}^{-1}$ is attributed to the doubly degenerate framework- $\mathrm{CH}_{3}$ bending mode. The methyl torsion seen here is anomalously high in energy; the methyl torsions in the $n$-alkanes all occur at $\sim 250 \mathrm{~cm}^{-1} .^{58,59}$ The unusually high energy does not appear to be a consequence of the crystal packing: ${ }^{30,41}$ the closest contacts are at $2.6 \AA$ and do not involve the methyl group.

It might be expected that the addition of more methyl groups in the fully methylated tribenzotriquinacene $\mathbf{1 0}$ would simply result in a corresponding number of very intense torsional modes. This is not the case; rather, a complex manifold of modes is observed (Fig. 10). The DFT calculations show that the four methyl torsions occur at: 239 (265), 277 (293) and $392(387) \mathrm{cm}^{-1}$ (experimental values in brackets). Visualisation of the modes provides two surprises: firstly, although the $293 \mathrm{~cm}^{-1}$ mode in the tetramethyl derivative $\mathbf{1 0}$ is at the same transition energy as for the monomethyl analogue $\mathbf{9}$, it does not involve the central methyl group, only the peripheral ones. It is a doubly degenerate mode, so its intensity is doubled, otherwise all of the modes would have comparable intensity. Secondly, the central methyl's torsion occurs at $387 \mathrm{~cm}^{-1}$ and, in contrast to the other torsional modes (which are almost pure torsions), it is highly mixed with the tribenzotriquinacene framework modes. A methyl torsion at this energy is completely unprecedented.

In the $\mathrm{C}-\mathrm{H}$ stretch region, the aromatic modes above $3000 \mathrm{~cm}^{-1}$ are very similar for all three tribenzotriquinacenes 3, 9 and 10. For the parent material, 3, with four methine $\mathrm{C}-\mathrm{H}$ groups, the classical methine stretch at $2901 \mathrm{~cm}^{-1}$ is seen, involving all four methines. In addition there is a band at $2976 \mathrm{~cm}^{-1}$ which is almost a pure C-H stretch of the central methine group. On substitution by the methyl group in hydrocarbon 9 , this vanishes and is replaced by weak modes at $2863 \mathrm{~cm}^{-1}\left(\mathrm{CH}_{3}\right.$ symmetric stretch) and $2924 / 2959 \mathrm{~cm}^{-1}$ $\left(\mathrm{CH}_{3}\right.$ asymmetric stretch). Complete substitution of the methine hydrogens in compound $\mathbf{1 0}$ by methyl groups results in the disappearance of the $2901 \mathrm{~cm}^{-1}$ band and the appearance of a cluster of bands in the regions expected for the symmetric and asymmetric methyl stretch modes. ${ }^{55}$

\section{Conclusions}

The vibrational spectra of a set of various centropolyindanes has provided insights into the bonding present and some surprises. The spectra clearly show that the ortho-phenylene rings of the indane sub-units largely behave as independent entities. There is no evidence for any significant coupling between them, as demonstrated by the similarity of the spectra, both to each other and to the parent molecule, indane (1). The $\mathrm{C}-\mathrm{C}$ stretch modes of the carbon atoms that are common to two or more indane sub-units occur in the usual range associated with $\mathrm{sp}^{3} \mathrm{C}-\mathrm{C}$ bonds, suggesting that these are conventional bonds.

The surprises occur on substitution of the molecules. Thus the carbonyl stretch mode of triptindan-9-one (7) is lower than would be expected. This is not a ring strain effect, but appears to be a longer-range effect of the additional ortho-phenylene rings. Methyl substitution in the tribenzotriquinacene derivatives $\mathbf{9}$ and $\mathbf{1 0}$ produces methyl torsional modes that are the highest known. The assignments provided here are a reference for future studies of these unusual hydrocarbons.

\section{Conflicts of interest}

There are no conflicts to declare.

\section{Acknowledgements}

The STFC Rutherford Appleton Laboratory is thanked for access to neutron beam facilities. Computing resources (time on the SCARF compute cluster for the CASTEP and Gaussian calculations) was provided by STFC's e-Science facility. This research has been performed with the aid of facilities at the Research Complex at Harwell $(\mathrm{RCaH})$, including the FT-Raman spectrometer. The authors would like to thank the Research Complex for access and support to these facilities and equipment.

\section{Notes and references}

$1 \mathrm{H}$. Hopf, Classics in Hydrocarbon Chemistry, Wiley-VCH, Weinheim, 2000.

2 A. Narita, X.-Y. Wang, X. Feng and K. Müllen, Chem. Soc. Rev., 2015, 44, 6616-6643.

3 E. L. Spitler, C. A. Johnson II and M. M. Haley, Chem. Rev., 2006, 106, 5344-5386.

4 V. M. Tsefrikas and L. T. Scott, Chem. Rev., 2006, 106, 4868-4884.

5 T.-Y. Wu and J. S. Siegel, Chem. Rev., 2006, 106, 4843-4867.

6 Y. Segawa, A. Yagi, K. Matsui and K. Itami, Angew. Chem., 2016, 128, 5222-5245 (Angew. Chem., Int. Ed., 2016, 55, 5136-5158).

7 K. Y. Cheung, X. Xu and Q. Miao, J. Am. Chem. Soc., 2015, 137, 3910-3914.

8 J.-W. Han, X. Li and H. N. C. Wong, Chem. Rec., 2015, 15, 107-131.

9 K. Ikemoto, J. Lin, R. Kobayashi, S. Sato and H. Isobe, Angew. Chem., 2018, 130, 8691-8695 (Angew. Chem., Int. Ed., 2018, 57, 8555-8559).

10 Fragments of Fullerenes and Carbon Nanotubes, ed. M. A. Petrukhina and L. T. Scott, Wiley, Hoboken, 2012.

11 C. Thilgen and F. Diederich, Chem. Rev., 2006, 106, 5049-5135. 
12 D. Wendinger and R. R. Tykwinski, Acc. Chem. Res., 2017, 50, 1468-1479.

13 P. Gund and T. M. Gund, J. Am. Chem. Soc., 1981, 103, 4458-4465.

14 Diamond and Related Nanostructures, Vol. 6 of Carbon Materials: Chemistry and Physics (F. Cataldo and P. Milani, Series $E d s$.$) , ed. M. V. Diudea and C. L. Nagy, Springer, Dordrecht,$ 2013.

15 M. Mastalerz, Acc. Chem. Res., 2018, 51, 2411-2422.

16 F. Beuerle and B. Gole, Angew. Chem., 2018, 130, 4942-4972 (Angew. Chem., Int. Ed., 2018, 57, 4850-4878).

17 X. Shen, D. M. Ho and R. A. Pascal Jr., Org. Lett., 2003, 5, 369-371.

18 D. Kuck, Chem. Rev., 2006, 106, 4885-4925.

19 H. W. Thompson, J. Org. Chem., 1968, 33, 621-625.

20 B. Paisdor and D. Kuck, J. Org. Chem., 1991, 56, 4753-4759.

21 D. Kuck, Angew. Chem., 1984, 23, 508-509 (Angew. Chem., Int. Ed. Engl., 1984, 96, 515-516).

22 D. Kuck, T. Lindenthal and A. Schuster, Chem. Ber., 1992, 125, 1449-1460.

23 G. Markopoulos, L. Henneicke, J. Shen, Y. Okamoto, P. G. Jones and H. Hopf, Angew. Chem., 2012, 124, 13057-13060 (Angew. Chem., Int. Ed., 2012, 51, 12884).

24 D. Kuck and H. Bögge, J. Am. Chem. Soc., 1986, 108, 8107-8109.

25 D. Kuck and A. Schuster, Angew. Chem., 1988, 100, 1222-1224 (Angew. Chem., Int. Ed. Engl., 1988, 27, 1192-1194).

26 H.-W. Ip, C.-F. Ng, H.-F. Chow and D. Kuck, J. Am. Chem. Soc., 2016, 138, 13778-13781.

27 W.-S. Wong, C.-F. Ng, D. Kuck and H.-F. Chow, Angew. Chem., 2017, 129, 12528-12532 (Angew. Chem., Int. Ed., 2017, 56, 12356-12360).

28 For example, see: D. Kuck, J. Linke, L. C. Teichmann, D. Barth, J. Tellenbröker, D. Gestmann, B. Neumann, H.-G. Stammler and H. Bögge, Phys. Chem. Chem. Phys., 2016, 18, 11722-11737.

29 C. R. Groom, I. J. Bruno, M. P. Lightfoot and S. C. Ward, Acta Crystallogr., Sect. B: Struct. Sci., Cryst. Eng. Mater., 2016, B72, 171-179.

30 D. Kuck, A. Schuster, R. A. Krause, J. Tellenbröker, C. P. Exner, M. Penk, H. Bögge and A. Müller, Tetrahedron, 2001, 57, 3587-3613.

31 S. F. Parker, F. Fernandez-Alonso, A. J. Ramirez-Cuesta, J. Tomkinson, S. Rudić, R. S. Pinna, G. Gorini and J. Fernández Castañon, J. Phys.: Conf. Ser., 2014, 554, 012003.

32 M. A. Adams, S. F. Parker, F. Fernandez-Alonso, D. J. Cutler, C. Hodges and A. King, Appl. Spectrosc., 2009, 63, 727-732.

33 M. J. Frisch, G. W. Trucks, H. B. Schlegel, G. E. Scuseria, M. A. Robb, J. R. Cheeseman, G. Scalmani, V. Barone, G. A. Petersson, H. Nakatsuji, X. Li, M. Caricato, A. Marenich, J. Bloino, B. G. Janesko, R. Gomperts, B. Mennucci, H. P. Hratchian, J. V. Ortiz, A. F. Izmaylov, J. L. Sonnenberg, D. Williams-Young, F. Ding, F. Lipparini, F. Egidi, J. Goings, B. Peng, A. Petrone, T. Henderson,
D. Ranasinghe, V. G. Zakrzewski, J. Gao, N. Rega, G. Zheng, W. Liang, M. Hada, M. Ehara, K. Toyota, R. Fukuda, J. Hasegawa, M. Ishida, T. Nakajima, Y. Honda, O. Kitao, H. Nakai, T. Vreven, K. Throssell, J. A. Montgomery Jr., J. E. Peralta, F. Ogliaro, M. Bearpark, J. J. Heyd, E. Brothers, K. N. Kudin, V. N. Staroverov, T. Keith, R. Kobayashi, J. Normand, K. Raghavachari, A. Rendell, J. C. Burant, S. S. Iyengar, J. Tomasi, M. Cossi, J. M. Millam, M. Klene, C. Adamo, R. Cammi, J. W. Ochterski, R. L. Martin, K. Morokuma, O. Farkas, J. B. Foresman and D. J. Fox, Gaussian 09, Revision D.02, Gaussian, Inc., Wallingford CT, 2016.

34 S. J. Clark, M. D. Segall, C. J. Pickard, P. J. Hasnip, M. J. Probert, K. Refson and M. C. Payne, Z. Kristallogr., 2005, 220, 567.

35 K. Refson, P. R. Tulip and S. J. Clark, Phys. Rev. B: Condens. Matter Mater. Phys., 2006, 73, 155114.

36 A. Tkatchenko and M. Scheffler, Phys. Rev. Lett., 2009, 102, 073005.

37 R. Dennington, T. A. Keith and J. M. Millam, GaussView, Version 5.0.9, Semichem Inc., Shawnee Mission, KS, 2016.

38 A. J. Ramirez-Cuesta, Comput. Phys. Commun., 2004, 157, 226-238.

39 N. M. O'Boyle, A. L. Tenderholt and K. M. Langner, J. Comput. Chem., 2008, 29, 839-845.

40 M. Harig, B. Neumann, H.-G. Stammler and D. Kuck, ChemPlusChem, 2017, 82, 1078-1095, see ref. 76 cited therein.

41 J. G. Brandenburg, S. Grimme, P. G. Jones, G. Markopoulos, H. Hopf, M. K. Cyranski and D. Kuck, Chem. - Eur. J., 2013, 19, 9930-9938.

42 M. I. Davis and T. W. Muecke, J. Phys. Chem., 1970, 74, 1104-1108.

43 S. F. Parker, D. Siegel, N. G. Hamilton, J. Kapitán, L. Hecht and D. Lennon, J. Phys. Chem. A, 2012, 116, 333-346.

44 J. F. Fuller and E. J. Valente, J. Chem. Crystallogr., 1996, 26, 815-821.

45 Y. Li, L. Zhang, Z. Zhang, J. Xu, Y. Pan, C. Xu, L. Liu, Z. Li, Z. Yu, H. Li and L. Xu, Adv. Synth. Catal., 2016, 358, 2148-2155.

46 L. B. Favero, W. X. Li, G. Spadini and W. Caminati, J. Mol. Spectrosc., 2015, 316, 45-48.

47 A. P. Scott and L. Radom, J. Phys. Chem., 1996, 100, 16502-16513.

48 T. L. Smithson, J. A. Duckett and H. Wieser, J. Phys. Chem., 1984, 88, 1102-1109.

49 K. H. Hassan and J. M. Hollas, J. Mol. Spectrosc., 1991, 147, 100-113.

50 Z. Arp, N. Meinander, J. Choo and J. Laane, J. Chem. Phys., 2002, 116, 6648-6655.

51 L. Bardet, G. Fleury, R. Granger and C. Sablayrolles, J. Mol. Struct., 1968, 2, 397-408.

52 G. Varsanyi, Assignments for vibrational spectra of 700 benzene derivatives, Akadémiai Kiadó, Budapest, 1973.

53 F. R. Dollish, W. G. Fateley and F. F. Bentley, Characteristic Raman frequencies of organic compounds, John Wiley \& Sons, New York, 1974. 
54 H. Jobic, S. Sportouch and A. Renouprez, J. Mol. Spectrosc., 1983, 99, 47-55.

55 D. Lin-Vien, N. B. Colthup, W. G. Fateley and J. G. Grasselli, The handbook of infrared and Raman characteristic frequencies of organic molecules, Academic Press, Boston, 1991.

56 D. S. Yufit and J. A. K. Howard, Acta Crystallogr., Sect. C: Cryst. Struct. Commun., 2011, 67, o104-0106.
57 T. P. Ruiz, M. Fernandez-Gomez, J. J. L. Gonzalez, A. E. Koziol and J. M. G. Roldan, J. Mol. Struct., 2004, 707, 33-46.

58 D. A. Braden, S. F. Parker, J. Tomkinson and B. S. Hudson, J. Chem. Phys., 1999, 111, 429-437.

59 J. Tomkinson, S. F. Parker, D. A. Braden and B. S. Hudson, Phys. Chem. Chem. Phys., 2002, 4, 716-721. 